\title{
Displacement Errors in Antenna Near-Field Measurements and Their Effect on the Far Field
}

\author{
LORANT A. MUTH
}

\begin{abstract}
The effects of probe displacement errors in the near-field measurement procedure on the far-field spectrum are studied. Expressions are derived for the displacement error functions that maximize the fractional error in the spectrum both for the on-axis and off-axis directions. The $x$-y and $z$-displacement errors in planar scanning are studied first and, consequently, the results are generalized to errors in spherical scanning. Some simple near-field models are used to obtain order of magnitude estimates for the fractional error as a function of relevant scale lengths of the near field, defined as the lengths over which significant variations occur.
\end{abstract}

\section{INTRODUCTION}

$\mathrm{T}$ HE UNAVOIDABLE ERRORS in the probe's position while scanning the near field of an antenna show up inevitably in the far field of the antenna being measured. As is well known [1], the far field of an antenna is obtained by taking the Fourier transform of the antenna's planar near field and performing additional algebraic manipulations to remove the effect of the receiving characteristics of the probe, a procedure known as probe correction. In evaluating the accuracy of a near-field range-planar, cylindrical or spherical-obvious and natural questions arise: 1) what systematic position errors will lead to maximum far-field errors; 2) what is the dependence of this maximum far-field error function on the wavenumber $\mathbf{k}$, whose magnitude is constant; and, more generally, 3) what is the exact error-contaminated far field of an antenna if its exact near field and an arbitrary probe displacement error function are known?

Previous studies raised the first two questions above [2]-[5] and treated them in the context of planar scanning. This paper reexamines the above question in the planar context from a slightly different point of view, with the intent to achieve enough generality in the mathematical formalism so that the analysis can be extended to study position errors in cylindrical and spherical scanning procedures. Some error expressions are derived in spherical geometry which can serve as the basis for computer simulation. Similar simulations in the planar case have been discussed in [3]. A general expression that answers the third question is also derived.

To accomplish the objectives of the paper, expressions for maximum systematic errors in all geometries have to be derived. First, simple general mathematical arguments are

Manuscript received June 18, 1987; revised September 16, 1987

The author is with the National Bureau of Standards, U.S. Department of Commerce, 325 Broadway, Boulder, CO 80303.

IEEE Log Number 8819824. used to get the structure and the relevant parameters that appear in the fractional error expressions; then a rigorous procedure for maximization of error is outlined for on-axis errors in mathematically real near fields. This simplified special case is considered first for a mathematical reason: the error expressions for realistic (complex) near fields for on- or off-axis directions in $k$-space can be obtained using the procedure worked out for the simplified on-axis case if a straightforward additional procedure is incorporated. Once this procedure is worked out, all special complicated cases such as steered beams and all errors in spherical geometry can be treated.

\section{General Mathematical Statement of the Problem}

We can derive general expressions for the fractional error in the spectrum of the near field $B(x)$ due to arbitrary position errors. Here $\mathbf{x}$ is an arbitrary three-dimensional position vector. If the real function $\delta \mathbf{x}(\mathbf{x})$ is the error in position of the probe at $\mathbf{x}$, then the near field measured is $B(\mathbf{x}+\delta \mathbf{x}(\mathbf{x}))$. The fractional error in the spectrum $D(\mathbf{k})$ due to position errors is then

$$
\frac{\Delta D(\mathbf{k})}{D(\mathbf{k})}=\frac{\int\{B(\mathbf{x}+\delta \mathbf{x}(\mathbf{x}))-B(\mathbf{x})\} e^{-i \mathbf{k} \cdot \mathbf{x}} d^{2} x}{\int B(\mathbf{x}) e^{-i \mathbf{k} \cdot \mathbf{x}} d^{2} x},
$$

where the integration is over the finite scan plane. The use of a finite plane of integration is an approximation in the denominator, but is exact in the numerator, since errors occur only at points where measurements are taken. In the above expression we also have $\mathbf{k}=(\mathbf{K}, \pm \gamma)$, where $\mathbf{K}=\left(k_{x}, k_{y}\right), k_{z}= \pm \gamma$ and $|k|=2 \pi / \lambda=$ constant for lossless media. We now seek the real function $\delta \mathbf{x}(\mathbf{x})$ that maximizes the fractional error in a given direction in $k$-space. The numerator in (1) will have a maximum for a finite $\delta \mathbf{x}(\mathbf{x})$ only if $\delta \mathbf{x}(\mathbf{x})$ is subject to the constraint

$$
\frac{1}{A} \int|\delta \mathbf{x}|^{2} d^{2} x=\sigma^{2}
$$

where $\sigma=$ constant, $A$ is the total scan area. Expressions (1) and (2) then define a variational problem wherein the function $\delta \mathbf{x}(\mathbf{x})$ that maximizes (1) can be found with the use of the functional derivative [6]. This simple procedure will be indicated below for the one-dimensional case. Physically, the constraint in (2) merely restricts the error functions to be 
considered to a constant root mean square (rms) value. Strictly speaking, the variational problem has to be formulated for either the real or imaginary part of (1) separately. To find the maximum of the absolute value of the fractional error, a slightly modified procedure has to be followed.

One can write down very general expressions for the maximum fractional error in (1) without having to specify $\delta \mathbf{x}(\mathbf{x})$. By the use of the mean value theorem for real functions [7] and for $\mathbf{K}=0$ (on axis), one can write (assuming that any $z$-dependence is specified as a function of $x$ and $y$, and suppressing the $k_{z}= \pm \gamma$ dependence)

$$
\mu \equiv \frac{\Delta D(0)}{D(0)}=\frac{B(\hat{\mathbf{x}}+\delta \mathbf{x}(\hat{\mathbf{x}}))-B(\hat{\mathbf{x}})}{B(\overline{\mathbf{x}})}
$$

where $\hat{\mathbf{x}}$ and $\overline{\mathbf{x}}$ are some points on the scan plane and $B(\overline{\mathbf{x}})$ is the average of the near-field measurements. We have assumed here that $\boldsymbol{B}(\mathbf{x})$ is essentially a real function (nonzero phase is allowed), since the mean value theorem cannot be applied to complex functions directly [7]. ${ }^{1}$ We can approximate (3) as

$$
\frac{\Delta D(0)}{D(0)}=\frac{|\nabla B||\delta \mathbf{x}|}{B(\overline{\mathbf{x}})}
$$

which in the one-dimensional case is (prime denotes differentiation)

$$
\frac{\Delta D(0)}{D(0)}=\frac{B^{\prime}(\hat{\mathbf{x}})|\delta \mathbf{x}(\hat{\mathbf{x}})|}{B(\overline{\mathbf{x}})} \leqslant \frac{B_{\max }^{\prime} \delta x_{\max }}{B(\overline{\mathbf{x}})} .
$$

As will be seen below, in a first order approximation a properly normalized displacement-error function has a maximum proportional to $\sigma$, hence (using $\alpha$ as constant of proportionality),

$$
\mu_{1} \equiv \frac{\Delta D(0)}{D(0)} \leqslant \alpha \sigma \frac{B_{\max }^{\prime}}{B(\overline{\mathbf{x}})}=c \frac{\sigma}{l}
$$

where $c$ is some constant of order unity, and $l$ is scale length of significant variation in the near field. In (5a) and below, the subscript on $\mu$ indicates the order of approximation. The exact value of $c$ can be obtained only from either an actual near field or a model of it. An order-of-magnitude estimate for $c$ can be obtained as follows: $B_{\max }^{\prime} \approx B_{\max } / l, B(\bar{x}) \approx B_{\max } / 2$ and assuming $\alpha \approx 1$, one obtains $c \approx 2$. As will be seen below, this is, indeed, a good estimate. (Equation (5a) agrees in form with [3, eq. (63a)].)

The scale length $l$ represents variations in the near field $B(x)$ either parallel or perpendicular to $\mathbf{k}_{0}$, the wave vector of interest. In case $\delta \mathbf{x}|| \mathbf{k}_{0}$, then $l^{-1} \approx\left|k_{0}\right|=2 \pi / \lambda$, as can be verified from the simple model of a plane wave of constant amplitude propagating in the direction $\delta \mathbf{x}$. Variations in the amplitude of the near field orthogonal to $\delta \mathbf{x}$ will be reflected in

\footnotetext{
${ }^{1}$ To reiterate, these simplifying assumptions are made here in order to develop an understanding of the relevant parameters and the structure of the error expressions sought. It is not true that this simple example is studied because more complicated cases cannot be treated. As will be seen below, the error analysis of any special case that is more general than the one considered here follows simply from the considerations in this section and the next, and the additional procedure outlined in Section III-B.
}

the constant in (5a), in this case denoted by $c_{\|}$. For $\delta \mathbf{x} \perp \mathbf{k}, l$ represents the scale length in the variation of the near-field profile along surfaces of constant phase. The constant in this case will be denoted by $c_{i}$. Hence, we can write

$$
\mu_{1}^{(\|)} \leqslant c_{\|} k \sigma_{\|}
$$

and

$$
\mu_{1}^{(\perp)} \leqslant c_{\perp} \frac{\sigma_{\perp}}{l}
$$

In the case of an on-axis beam (K=0), (5b) represents the upper bound error resulting from $z$-displacement errors (out of the scan plane), and (5c) represents the upper bound resulting from displacement errors in the scan plane. The ratio

$$
\frac{\mu^{(\perp)}}{\mu^{(\|)}} \approx \frac{\lambda}{l} \ll 1
$$

in general.

Two alternative expressions to (4) can be shown to be, using $\beta$ for the unit amplitude near field and denoting derivatives by a prime

$$
\left|\frac{\Delta D(0)}{D(0)}\right| \leqslant \sigma \frac{\beta_{\max }^{\prime}}{\langle\beta\rangle} \frac{\beta_{\max }^{\prime}}{\left\langle\beta^{\prime 2}\right\rangle^{1 / 2}},
$$

and

$$
\left|\frac{\Delta D(0)}{D(0)}\right|^{2} \leqslant \sigma^{2} \frac{\left\langle\left[\beta^{\prime}(x)\right]^{2}\right\rangle}{\langle\beta(x)\rangle^{2}},
$$

where the $\langle\cdot\rangle$ implies the average. These expressions will be derived in the next section. Again, the derivatives in (6) and (7) represent directions either parallel or orthogonal to $\mathbf{k}$. Here the unit amplitude near field has been introduced to emphasize the fact that the absolute magnitude of the near field cancels out in these expressions.

In (5)-(7) it has been assumed that the near field is real. If the fractional error in directions other than $\mathbf{K}=0$ is desired or if the near field is complex (i.e., for any real antennas, steered beams or electrically small antennas), the derivations of scalelength expressions are slightly more complicated, but fundamentally present no great difficulties.

\section{The Maximization Procedure}

To solve the variational problem as stated in (1) and (2) in complete generality, we have to proceed in steps. First, we solve the simplified problem, where the near field is a real function and the wave vector in the exponential vanishes. Then in the one-dimensional case we seek to maximize the integral

$$
I=\int_{L}[B(x+\delta x)-B(x)] d x,
$$

subject to the constraint

$$
\frac{1}{L} \int_{L}(\delta x)^{2} d x=1
$$

where $L$ is the interval of interest. We use the Lagrange 
multiplier $\eta[6]$ to maximize

$$
\hat{I}=\int_{L}[B(x+\delta x)-B(x)] d x-\eta \int_{L}(\delta x)^{2} d x
$$

with respect to $\delta x$. Thus,

$$
\frac{\delta \hat{I}}{\delta(\delta x)}=\int_{L}\left[B^{\prime}(x+\delta x)-2 \eta \delta x\right] d x=0
$$

or

$$
\delta x=\frac{1}{2 \eta} B^{\prime}(x+\delta x) .
$$

Equation (10c) is an implicit statement specifying $\delta x(x)$ that will maximize the integral in (8). To obtain $\delta x(x)$ explicitly, (10c) is expanded in a Taylor series and the constant $\eta$ is obtained from (9). The conditions for first- and higher order approximation values can thus be worked out. If we want the displacement-error function to satisfy

$$
\frac{1}{L} \int_{L}(\delta x)^{2} d x=\sigma_{x}^{2} \neq 1
$$

instead of condition (9), we merely have to multiply $\delta x$ in (9) by $\sigma_{x}$. Thus there is no loss of generality in using (11), as (9) is a special case of (11), i.e., the maximization procedure is independent of $\sigma_{x}$, which appears only as a linear parameter of the problem.

The Taylor series expansion of $(10 \mathrm{c})$ is

$$
\delta x=\frac{1}{2 \eta}\left\{B^{\prime}(x)+B^{\prime \prime}(x) \delta x+\frac{1}{2 !} B^{\prime \prime \prime}(x)(\delta x)^{2}+\cdots\right\} .
$$

For a first-order expansion in $\delta x$ the condition

$$
\left|\frac{1}{2 !} \frac{B^{\prime \prime \prime}(x)}{B^{\prime \prime}(x)}(\delta x)\right| \ll 1
$$

must hold, and similarly for higher order terms. This implies

$$
\delta x=\frac{B^{\prime}(x)}{2 \eta-B^{\prime \prime}(x)}
$$

where $\eta$ is determined by (11). If we further assume that

$$
B^{\prime \prime}(x) \ll 2 \eta
$$

then from (13) and (11)

$$
\frac{1}{4 \eta^{2} L} \int_{L}\left[B^{\prime}(x)\right]^{2} d x=\sigma_{x}^{2}
$$

and

$$
\delta x \cong \sigma_{x} \frac{B^{\prime}(x)}{\left\langle B^{\prime 2}\right\rangle^{1 / 2}}
$$

satisfies (11). Thus, the displacement error function leading to maximum far-field errors has been found. Consistent with the first-order approximation developed here, the integral in (8) is approximated as

$$
I \cong \int_{L} B^{\prime}(x) \delta x d x
$$

with

$$
\left|\frac{1}{2 !} \frac{B^{\prime \prime}(x)}{B^{\prime}(x)} \delta x\right| \ll 1
$$

and similarly for higher order terms. Using (15), (12) becomes

$$
\left|\frac{\sigma_{x}}{2} \frac{B^{\prime \prime}(x)}{B^{\prime \prime}(x)} \frac{B^{\prime}(x)}{\left\langle B^{\prime 2}\right\rangle^{1 / 2}}\right| \ll 1
$$

and (14) and (18) are consistently satisfied by

$$
\left|\sigma_{x} \frac{B^{\prime \prime}(x)}{\left\langle B^{\prime 2}\right\rangle^{1 / 2}}\right| \ll 1 .
$$

It is easy to see that (19a) and (19b) can be satisfied for a small enough $\sigma_{x}$. Special attention must be given to points where $B^{\prime \prime}(x)=0$, but we will not address that here.

The maximum of (17) is obtained using (15)

$$
I \cong \sigma_{x} L\left\langle B^{\prime 2}\right\rangle^{1 / 2}
$$

and the fractional error in (1) can be written as in (7)

$$
\mu_{1}^{2}=\left|\frac{\Delta D(0)}{D(0)}\right|^{2} \leqslant \sigma_{x}^{2} \frac{\left\langle B^{\prime 2}\right\rangle}{\langle B(x)\rangle^{2}}=\sigma_{x}^{2} \frac{\left\langle\beta^{\prime 2}\right\rangle}{\langle\beta(x)\rangle^{2}}
$$

where $\beta(x)$ is the unit-amplitude near field. Higher order approximation schemes to solve (8) and (10) consistently can be worked out, but this will not be done here, since the unwieldy algebraic manipulations lead to no new results. In (1) and (2) a second-order expansion of the integral in (8) has been found useful, i.e., the maximization of

$$
\int\left\{B^{\prime}(x) \delta x+\frac{1}{2 !} B^{\prime \prime}(x)(\delta x)^{2}\right\} d x
$$

has been sought. We can use the first-order expression (15) to get

$$
\mu_{2} \leqslant \frac{1}{\langle\beta\rangle}\left[\sigma_{x}\left\langle\beta^{\prime 2}\right\rangle^{1 / 2}+\frac{1}{2 !} \sigma_{x}^{2} \frac{\left\langle\beta^{\prime \prime} \beta^{\prime 2}\right\rangle}{\left\langle\beta^{\prime 2}\right\rangle}\right] .
$$

The more exact expression for $\mu_{2}$ using a second-order expansion in (10) would result in a much more complicated form. Again the amplitude of the near field is seen to cancel; hence the use of $\beta$ in (21b).

Since $\Delta D=D_{e}-D_{0}$, where $D_{e}$ is the error-contaminated spectrum, one can write $D_{e}=D_{0}\left(1+\mu_{1}\right)$ or

$$
R(\mathrm{~dB}) \equiv 20 \log _{10}\left(\frac{D_{e}}{D_{0}}\right) \approx 8.7 \mu_{1} .
$$

This result together with (21a) can be compared to that given in [3] where an equation of the same form has been derived. Before proceeding to maximize (8) for complex near fields $B(x)$ or for $\mathbf{K} \neq 0$ (off axis), a few elementary near-field 
models will be used to exhibit some explicit results for the fractional error $\mu$.

\section{A. Some Simple Models}

Two basic models that incorporate the most essential features of near fields will be used in expressions (21) and

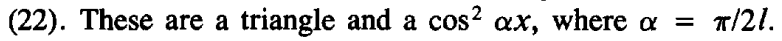
These are illustrated in Fig. 1. Both of these models have a scale length $l$. The model independence of the maximization process can be surmised by deriving results for both of these models. For the triangle $\left|\beta^{\prime}\right|=(1 / l) \beta^{\prime \prime}=0$, except at $x=$ 0 , and $\langle\beta\rangle=1 / 2(2 l)(1) / 2 l=1 / 2$. So

$$
\mu_{1} \leqslant 2 \frac{\sigma_{x}}{l}
$$

The second-order term in $\mu_{2}$ contributes only at $x=0$, which cannot be calculated by elementary means. If we approximate $\left\langle\beta^{\prime \prime}\right\rangle \approx-1 / l^{2}$, then

$$
\mu_{2} \leqslant 2 \frac{\sigma_{x}}{l}-\left(\frac{\sigma_{x}}{l}\right)^{2} .
$$

For $\beta=\cos ^{2} \alpha x, \beta^{\prime}=-\alpha \sin 2 \alpha x, \beta^{\prime \prime}=-2 \alpha^{2} \cos 2 \alpha x$, and

$$
\mu_{1} \leqslant \frac{\pi}{\sqrt{2}} \frac{\sigma_{x}}{l} \approx 2.22 \frac{\sigma_{x}}{l} .
$$

In this case, there is no contribution from the second-order term. However, in general, asymmetric near fields will contribute here and will be of the form as in (23b). In practice this will arise for steered beams.

Comparing (23a) and (23c) one can see that the results are essentially model independent, since the constant coefficients are essentially equal and the other parameters enter exactly the same way.

On closer examination, it is found that for this example (19b) gives the most stringent condition on $\sigma_{x}$, i.e.,

$$
\sigma_{x}^{2} \ll \frac{1}{2}\left(\frac{l}{\pi}\right)^{2}
$$

must hold for the analysis presented in Section III to be valid. If $L \equiv 2 l$ is the aperture dimension, then $\sigma_{x} \ll L / 9$. Let $L=$ $n \lambda$, where $\lambda$ is the wavelength and $n$ is some constant, then $\sigma_{x} \ll m(0.1 \lambda)$, where $m \approx 1.13$. In practice, such a rootmean-square position error is attainable. Using (22), one can write

$$
R(\mathrm{~dB}) \simeq 40 \frac{\sigma_{x}}{L}
$$

or

$$
\frac{\sigma_{x}}{\lambda} \simeq \frac{1}{40} n R
$$

This last relationship gives the rms displacement error in units of wavelengths in terms of $R$, the far-field error in decibels, and $n$, the aperture size, in units of wavelength.

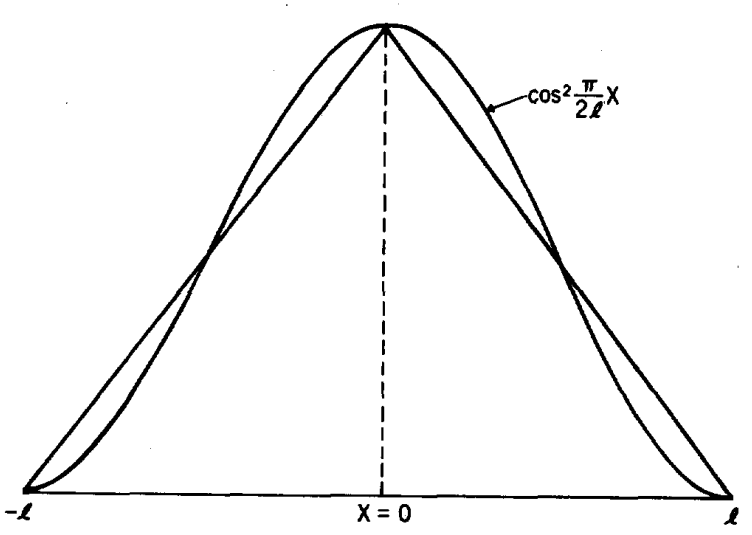

Fig. 1. Simple models for the near field to calculate the effects of probe displacement errors.

\section{B. Maximization of General Complex Near-Field Error Integral}

For general complex near fields or for errors in the off-axis direction, the expression whose amplitude is to be maximized has both real and imaginary parts; i.e.,

$$
I=\int G_{r}(x, \delta x(x)) d x+i \int G_{i}(x, \delta x(x)) d x .
$$

$G_{r}\left(G_{i}\right)$ is the real (imaginary) part of the integrand in the numerator of (1). One can either maximize the real (imaginary) part using the same procedure as for on-axis real near fields, but the maximum of the amplitude of $I$ in (26) will not, in general, be thus attained. Only if

$$
\int g_{r} g_{i} d x=0
$$

where $g_{r}=\left[G_{r}\right]^{\prime}, g_{i}=\left[G_{i}\right]^{\prime},[Q]^{\prime}=\partial Q^{\prime} /\left.\partial(\delta x)\right|_{\delta x=0}$, will the maximum of $I$ be given by the larger of the maxima of the two integrals in (26). The proof of this simple fact will not be detailed here. If condition (27) does not hold, we look for the function that will maximize (26) as a linear combination of functions that maximize each of the integrals separately. Thus,

$$
\delta x=\alpha \delta x^{(r)}+\beta \delta x^{(i)}
$$

where $\delta x^{(r)}\left(\delta x^{(i)}\right)$ is the displacement error function which maximizes the real (imaginary) part of the (26), and $\alpha$ and $\beta$ are constants determined by conditions (31) and (32) below. Adapting the results in Section III, a first-order approximation scheme to maximize (26) is as follows:

Let

$$
\begin{gathered}
\delta x^{(r)}=g_{r} \\
\delta x^{(i)}=g_{i} \\
I_{r}=\int g_{r}^{2} d x \\
I_{i}=\int g_{i}^{2} d x \\
I_{m}=\int g_{r} g_{i} d x .
\end{gathered}
$$


Then expanding (26) for small $\delta x(x)$ and from (11) and (28)

$$
\begin{gathered}
|I|^{2}=\left(\alpha I_{r}+\beta I_{m}\right)^{2}+\left(\alpha I_{m}+\beta I_{i}\right)^{2} \\
2 l \sigma_{x}^{2}=\alpha^{2} I_{r}+2 \alpha \beta I_{m}+\beta^{2} I_{i}
\end{gathered}
$$

and one determines the parameters $\alpha$ and $\beta(\alpha)$ from

$$
\begin{aligned}
& \frac{d|I|^{2}}{d \alpha}=0 \\
& \frac{d\left(\alpha_{x}^{2}\right)}{d \alpha}=0 .
\end{aligned}
$$

The details of this calculation are presented in Appendix I, where final expression for $\alpha$ and $\beta$ are derived as well as conditions that must hold for the special cases $\alpha \neq 0, \beta=0$ and $\alpha=0, \beta \neq 0$ to maximize (29) subject to constraint (30).

A more detailed treatment of a specific case of (26) is given in Section III-D and Appendix II.

\section{Maximization of Off-Axis Errors $(\mathbf{K} \neq 0)$}

If in the region of interest the near field is real, the off-axis error displacement function

$$
\delta x=\frac{B^{\prime}(x)\left\{\begin{array}{l}
\sin k x \\
\cos k x
\end{array}\right\}}{2 \eta-B^{\prime \prime}\left\{\begin{array}{l}
\sin k x \\
\cos k x
\end{array}\right\}}
$$

will maximize the integral

$$
\int B^{\prime}(x) \delta x\left\{\begin{array}{c}
\sin k x \\
\cos k x
\end{array}\right\} d x
$$

where $\eta$ is determined by (11). If (27) holds, i.e.,

$$
\left.\int B^{\prime}(x)\right]^{2} \cos k x \sin k x d x=0
$$

either integral with $\sin k x(\cos k x)$ will maximize the corresponding fractional error $\mu$. For near fields symmetric about the origin (35) will be satisfied. In practice, most near fields have a small asymmetric component, so (35) is only approximately satisfied. In case the asymmetry is significant, the procedure outlined in Sections III-B, III-D, and in Appendix II has to be followed. The results for $|D(k)| \neq 0$ are

$$
\mu_{1}^{2} \equiv\left|\frac{\Delta D(k)}{D(k)}\right|^{2} \leqslant \sigma_{x}^{2} \frac{\left\langle B^{\prime 2} \sin ^{2} k x\right\rangle}{\langle B(x) \cos k x\rangle^{2}+\langle B(x) \sin k x\rangle^{2}}
$$

and

$$
\left|\frac{\Delta D(k)}{D(0)}\right|^{2} \leqslant \sigma_{x}^{2} \frac{\left\langle B^{\prime 2} \sin ^{2} k x\right\rangle}{\langle B(x)\rangle^{2}}
$$

and similarly for $\cos k x$. Similar calculations to obtain secondorder corrections could be easily performed.

In Appendix III, (37) is evaluated for the simple model $B(x)$ $=\cos ^{2} \alpha x$ in Fig. 1, and the results are compared to real simulations.

\section{Steered Beams}

In the case of steered beams, displacement errors both in the scan plane and perpendicular to the scan plane have components along the off-axis beam direction. One can model such a beam to zeroth order by the near field

$$
B(x)=b(x) e^{i \epsilon x}
$$

where $\epsilon$ is some wavenumber and $b(x)$ is one of the profiles depicted in Fig. 1. Mathematically, the problem of maximizing the error integral (26) (either for $\mathbf{k}=\epsilon$, or $\mathbf{k} \neq \epsilon$ ) can be simplified if one keeps in mind the ratio in (5d); i.e., if the beam is steered enough off axis so that displacement errors in the scan plane correspond geometrically to displacement errors parallel to $\mathbf{k}$, with a small additional effect due to errors perpendicular to $\mathbf{k}$. If the beam angle is $\theta$, a first-order approximation is

$$
\mu_{1} \approx c_{\|} \sigma_{\|}|\epsilon|\left\{\begin{array}{c}
\cos \theta \\
\sin \theta
\end{array}\right\}+c_{\perp} \frac{\sigma_{\perp}}{l}\left\{\begin{array}{c}
\sin \theta \\
\cos \theta
\end{array}\right\}
$$

where, depending on the magnitude of $\theta$, one of the terms is negligible compared to the other. The choice of the trigonometric function depends on whether one is examining $z$ or $x-y$ displacement errors. For example, for $\theta=0$, only the term $c_{\|} \sigma_{\|}|\epsilon|$ contributes for $z$ errors, and for $x-y$ errors only the term $c_{\perp} \sigma_{\perp} / l$ contributes. For $\theta=\pi / 2$ similar reasoning shows that the role of each term in (39) is interchanged. For angles such that any error has a significant projection both parallel and perpendicular to $\mathbf{k},(39)$ is essentially valid, but the full analysis is outlined in Section III-B and Appendix I has to be carried out to determine the constants.

For $\mathbf{k} \neq \epsilon$, the expressions in Section III-C can be easily adopted for beams steered sufficiently off axis. One merely has to put the symbols in that section as

$$
\begin{gathered}
k \rightarrow \epsilon-k \\
B(x) \rightarrow b(x) \\
B^{\prime}(x) \rightarrow \epsilon b(x)
\end{gathered}
$$

and

$$
k=0 \rightarrow k=\epsilon .
$$

The adaptation of the maximization procedure for steered beams is detailed in Appendix II.

\section{The ERror-Contaminated Far Field}

The error-contaminated spectrum of the near field can be calculated exactly if the near field of the antenna and the exact displacement-error function are known. Let $D_{e}(\mathbf{K})$ be the error-contaminated far field

$$
D_{e}(\mathbf{K})=\int B(\mathbf{x}+\delta \mathbf{x}(\mathbf{x})) e^{-i \mathbf{k} \cdot \mathbf{x}} d^{2} x
$$


which, for errors in the $x$-direction only, can be expanded as

$$
\begin{aligned}
D_{e}(\mathbf{K})=\int[B(x)+ & \frac{\partial}{\partial x} B(x) \delta x \\
& \left.+\frac{1}{2 !} \frac{\partial^{2}}{\partial x^{2}} B(x)(\delta x)^{2}+\cdots\right] e^{-i \mathbf{k} \cdot \mathbf{x}} d^{2} x .
\end{aligned}
$$

The general expansion is a three-dimensional Taylor series. Since

$$
B(\mathbf{x})=\int D\left(\mathbf{K}^{\prime}\right) e^{i \mathbf{k}^{\prime} \cdot \mathbf{x}} d^{2} K^{\prime}
$$

spatial derivatives of $B(x)$ can be obtained from (42). Each differentiation will introduce a factor of $i k_{j}, j=1,2,3$ into the integrand of (41) with the result that

$$
D_{e}(\mathbf{K})=\int D\left(\mathbf{K}^{\prime}\right)\left[\int e^{i\left(\mathbf{k}^{\prime}-\mathbf{k}\right) \cdot \mathbf{x}} e^{i \mathbf{k} \cdot \delta \mathbf{x}(\mathbf{x})} d^{2} x\right] d^{2} K^{\prime}
$$

gives the error contaminated spectrum in terms of the known exact spectrum and the known displacement error function. Equation (43) can be written as

$$
D_{e}(\mathbf{K})=\int D\left(\mathbf{K}^{\prime}\right) E\left(\mathbf{K}^{\prime}, \mathbf{K}\right) d^{2} K^{\prime}
$$

where

$$
E\left(\mathbf{K}^{\prime}, \mathbf{K}\right)=\int e^{i\left(\mathbf{k}^{\prime}-\mathbf{k}\right) \cdot \mathbf{x}} e^{i \mathbf{k}^{\prime} \cdot \delta \mathbf{x}(\mathbf{x})} d^{2} x
$$

If $\delta \mathbf{x}(\mathbf{x}) \equiv 0$, then $E\left(K^{\prime}, K\right)=\delta\left(\mathbf{k}^{\prime}-\mathbf{k}\right)$ and $D_{e}(\mathbf{K})=$ $D(\mathbf{K})$; i.e., there is no error in the spectrum. If $\delta \mathbf{x}(\mathbf{x}) \equiv \mathbf{c}$, the result is essentially the same, as merely a phase factor $e^{i \mathbf{k} \cdot \mathbf{c}}$ is introduced. Another simple special case follows if $\delta \mathbf{x}(\mathbf{x})=$ $\left(\alpha_{1} x, \alpha_{2} y\right)$ as discussed in [3].

For small $\delta \mathbf{x}(\mathbf{x})$ such that $\mathbf{k} \cdot \delta \mathbf{x} \ll 1,(43)$ immediately yields a first-order approximation

$$
D_{e}(\mathbf{K})=\int D\left(\mathbf{K}^{\prime}\right) E_{1}\left(\mathbf{K}^{\prime}, \mathbf{K}\right) d^{2} K^{\prime}
$$

where

$$
E_{1}\left(\mathbf{K}^{\prime}, \mathbf{K}\right)=\delta\left(\mathbf{K}-\mathbf{K}^{\prime}\right)+\int e^{i\left(\mathbf{k}^{\prime}-\mathbf{k}\right) \cdot \mathbf{x}} i \mathbf{k} \cdot \delta \mathbf{x} d^{2} x .
$$

If we model $\delta \mathbf{x}$ as a sum of delta functions, i.e., $\delta \mathbf{x}(\mathbf{x})=$ $\Sigma_{n} \epsilon_{n} \delta\left(\mathbf{x}-\mathbf{x}_{n}\right)$ where $\epsilon_{n}$ is a vectorial amplitude and $\mathbf{x}_{n}$ are the grid points where measurements are taken, then (44b) yields

$$
\begin{aligned}
\Delta D(\mathbf{K}) \equiv D_{e}(\mathbf{K})-D(\mathbf{K}) & =i \mathbf{K} \\
& \cdot\left(\sum_{n} \epsilon_{n} B\left(\mathbf{x}_{n}\right) e^{-i \mathbf{k} \cdot \mathbf{x}_{n}}\right) .
\end{aligned}
$$

Here, $\epsilon_{n}$ is an unrestricted amplitude of the displacement function, and hence could be a random number. In such an event, (44c) gives the effects of random displacement errors on the far field.

\section{Displacement Errors in Spherical scanning}

It has been observed experimentally that for electrically large antennas the near-field amplitudes obtained in planar and spherical scanning are essentially equal [3]. Thus,

$$
\left|B_{s}(\mathbf{r})\right| \approx\left|B_{p}(\mathbf{x})\right|
$$

where $B_{s}(\mathbf{r})$ is the spherical and $B_{p}(\mathbf{x})$ is the planar near field and $\mathbf{r}$ is the general three-dimensional position vector. The phases across the main beam, however, differ, primarily due to the change in the $z$ displacement between the probe and the antenna. In a planar scan, the phase is essentially constant, but in the spherical scan the significant phase is given by

$$
\begin{gathered}
k \Delta z(x) \approx \pi\left(\frac{R}{\lambda}\right)\left(\frac{x}{R}\right)^{2}, y=0 \\
\left(\frac{x}{R}\right) \ll 1
\end{gathered}
$$

where $R$ is the radius of the scan sphere and $x^{2}+z^{2}=R^{2}$ is the intersection of the scan sphere and the $y=0$ plane.

The simple expressions in (45) and (46) can be exploited in spherical error analysis to take advantage of the results obtained in planar error analysis: one merely has to transform the phase of $\boldsymbol{B}_{s}(\mathbf{r})$ according to (46) and approximate $\boldsymbol{B}_{p}(\mathbf{x})$ with $B_{s}(\mathbf{r})$. The additional effect due to the variation of the orientation of the probe in spherical scanning as a function of position relative to the constant orientation of the probe in planar scanning is only significant at extreme angles and is neglected in this section. Accordingly, errors in spherical scanning will, in general, be a linear combination of $z$ and $x-y$ errors in planar scanning. The linear coefficients will depend on an averaged geometric relationship between the sphere and the plane, as will now be shown.

Consider a displacement error function $\delta \theta(\theta)$ along an arc that projects onto the $x$ - and $z$-axis at $y=0$. The $x$ - and $z$ components of $\delta \theta$ are

$$
\delta \theta(\theta)=\delta \theta(\theta)[(\hat{\theta} \cdot \hat{x}) \hat{x}+(\hat{\theta} \cdot \hat{z}) \hat{z}]
$$

or

$$
R \delta \theta(\theta)=\delta \theta(\theta)[R \cos \theta \hat{x}-R \sin \theta \hat{z}] .
$$

In planar notation, (47) is

$$
\begin{gathered}
\delta x(x)=\delta \theta(x) R \cos \theta=z \delta \theta(x) \\
\delta z(x)=-\delta \theta(x) R \sin \theta=-x \delta \theta(x)
\end{gathered}
$$

were $\theta=\sin ^{-1}(x / R)$, and $R$ is the scan radius. Here $L, R$, and $\theta_{\max }$, the maximum scan angle, are related by $\theta_{\max }=\sin ^{-1}$ $(L / R)$. Both displacement error functions in (48) must be taken into account in the maximization procedure, and Section III must be altered accordingly.

The constraint (corresponding to (11)) is now written as

$$
\frac{1}{R \Delta \theta} \int_{L}(R \delta \theta)^{2} \frac{R d \theta}{d x} d x=\sigma_{\theta}^{2}
$$

where $d \theta / d x=1 / z$. The expression to be maximized is now

$$
\hat{I}=\int_{L}\{B(x+R \delta \theta(x))-B(x)\} d x-\eta R \int_{L} \frac{(R \delta \theta)^{2}}{z} d x
$$


which to first order is

$$
\hat{I}=\frac{1}{R} \int_{L}\left\{\frac{\partial B}{\partial x} z-\frac{\partial B}{\partial z} x\right\}(R \delta \theta) d x-\eta R \int_{L} \frac{(R \delta \theta)^{2}}{z} d x
$$

The functional derivative of $\hat{I}$ in (51) with respect to $(R \delta \theta(x))$ will give the maximizing function similar to (15). Since $\partial B / \partial z$ $=i \gamma B$, the integrand in (51) is complex, in general, and the procedure outlined in Section III-B must be followed. Each term is maximized separately by

$$
R \delta \theta(x)=\frac{1}{2 \eta}\left(\frac{z}{R}\right)^{2} \frac{\partial B}{\partial x},
$$

and

$$
R \delta \theta(x)=\frac{1}{2 \eta}\left(\frac{z}{R}\right)\left(\frac{x}{R}\right) \frac{\partial B}{\partial z}
$$

Hence,

$$
R \delta \theta(x)=\sigma_{\theta} \frac{\left(\frac{z}{R}\right)^{2} B_{x}(x)}{\left|\left(\frac{z}{R}\right)^{3}\left(B_{x}\right)^{2}\right|_{\theta}^{1 / 2}}
$$

where $B_{x}=\partial B / \partial x$ and one defines $\langle Q\rangle_{\theta} \equiv(1 / R \Delta \theta) \int_{L} Q d x$, where $Q$ is any quantity. Similarly, for $z$-displacements

$$
R \delta \theta(x)=\sigma_{\theta} \frac{\left(\frac{z}{R}\right)\left(\frac{x}{R}\right) B_{z}(x)}{\left|\left(\frac{z}{R}\right)\left(\frac{x}{R}\right)^{2}\left(B_{z}\right)^{2}\right|_{\theta}^{1 / 2}}
$$

The two integrals in (51) become

$$
\hat{I}=\sigma_{\theta} R \Delta \theta\left\langle\left.\left(\frac{z}{R}\right)^{3}\left(B_{x}\right)^{2}\right|_{\theta} ^{1 / 2}\right.
$$

and

$$
\hat{I}=\sigma_{\theta} R \Delta \theta\left|\left(\frac{z}{R}\right)\left(\frac{x}{R}\right)^{2}\left(B_{z}\right)^{2}\right|_{\theta}^{1 / 2}
$$

and the corresponding on-axis fractional errors are

$$
\mu_{1}^{2}(0) \leqslant \sigma_{\theta}^{2} \frac{\left\langle\left(\frac{z}{R}\right)^{3}\left(B_{x}\right)^{2}\right\rangle}{\langle B(x)\rangle^{2}}
$$

or

$$
\mu_{1}^{2}(0) \leqslant \sigma_{\theta}^{2} \frac{\left\langle\left(\frac{z}{R}\right)\left(\frac{x}{R}\right)^{2}\left(B_{z}\right)^{2}\right\rangle}{\langle B(x)\rangle^{2}}
$$

Expressions (55)-(58) can be compared to (20) and (21). The presence of the geometric factors $(z / R)$ and $(x / R)$ in these new expressions merely reflect the fact that the magnitude of $\delta \theta(x)$ is being optimized rather than the displacements in the $x$ $y$ plane.

Equations (52) and (58) individually maximize the respective terms in (51). However, as we have seen in Section III-B, the maximization of $|\hat{I}|$ in (50) is given by the linear combination

$$
R \delta \theta(x)=\alpha\left(\frac{z}{R}\right)^{2} \frac{\partial B}{\partial x}+\beta\left(\frac{z}{R}\right)\left(\frac{x}{R}\right)\left|\frac{\partial B}{\partial z}\right|
$$

where $\alpha$ and $\beta$ are determined using the method outlined in Section III-B and Appendix I.

The corresponding treatment for radial displacement errors is outlined in Appendix IV.

\section{A. A Simple Model in Spherical Geometry}

In general, the on-axis fractional errors are essentially (from (57) through (58))

$$
\begin{aligned}
\mu_{1}^{2}(0) \leqslant c_{x}^{2}\left(\frac{\sigma_{\theta}}{l}\right)^{2}\left(\frac{\bar{z}}{R}\right)^{3} & +c_{z}^{2} \sigma_{\theta}^{2} k^{2}\left(\frac{\hat{z}}{R}\right)\left(\frac{\hat{x}}{R}\right)^{2} \\
& +c_{x z}^{2} \sigma_{\theta}^{2}\left(\frac{k}{l}\right)\left(\frac{\tilde{z}}{R}\right)^{2}\left(\frac{\tilde{x}}{R}\right)
\end{aligned}
$$

where $\tilde{z}, \bar{z}, \hat{z}, \hat{x}$, and $\tilde{x}$ are some intermediate values in the range $(0, R)$. For narrow-beam antennas $l \gg \lambda$, the second and third terms predominate, in that order. The simple models in figure 1 can be used to estimate in somewhat more detail the terms in (57), (58), and (60). These estimates are, to leading order, for $\theta_{\max } \neq 0$,

$$
\begin{array}{ll}
\frac{3}{2}\left(\frac{\sigma}{R}\right)^{2} \frac{\theta_{\max }}{\sin ^{3} \theta_{\max }}, & \text { for } x \text {-component } \\
\frac{1}{4} \sigma^{2} k^{2} \frac{\theta_{\max }}{\sin ^{3} \theta_{\max }}, & \text { for } z \text {-component }
\end{array}
$$

and

$$
\frac{4}{3} \sigma^{2} \frac{k}{l} \sin ^{3} \theta_{\max }, \quad \text { for the mixed component. (61c) }
$$

These expressions are comparable to (5b) and (5c) and to (23) and (25).

\section{Summary}

The effects of probe displacement errors on the far-field spectrum have been examined both for planar and spherical scanning. Expressions for the displacement errors that maximize the error in the far field have been derived using a method well known in the calculus of variations. The treatment of the planar case is straightforward, but the spherical problem is complicated by the fact that an error in a spherical coordinate corresponds to both $x-y$ and $z$ errors in planar geometry. Hence, a more complicated maximization procedure had to be adopted after the spherical data were 
transformed both in amplitude and phase onto the plane. To first order all fractional errors can be expressed as functions of $c(\sigma / l)$, where $c$ is some constant of order unity, $l$ is the relevant length scale either parallel or orthogonal to the direction in $k$-space under observation, and $\sigma$ is an integral measure (constraint) of the total mean-square error of the measuring system.

\section{APPENDIX I}

Maximization of General Complex Near-Field Error INTEgrals: SOME Further Detalls

In Section III-B, the maximization of general complex nearfield error integrals has been outlined. Here we present some important details. All quantities used in this Appendix have been previously defined in Section III-B.

Condition (32) results in

$$
\frac{d \beta}{d \alpha}=-\frac{\alpha I_{r}+\beta I_{m}}{\alpha I_{m}+\beta I_{i}},
$$

and condition (31) gives

$$
\frac{d \beta}{d \alpha}=\frac{I_{m}+I_{i} \frac{d \beta}{d \alpha}}{I_{r}+I_{m} \frac{d \beta}{d \alpha}} .
$$

Equation (62a) leads to

$$
\alpha=-\beta \frac{I_{m}+\frac{d \beta}{d \alpha} I_{i}}{I_{r}+\frac{d \beta}{d \alpha} I_{m}},
$$

and $(62 \mathrm{~b})$ leads to

$$
\left(\frac{d \beta}{d \alpha}\right)^{2}+\frac{d \beta}{d \alpha}\left[\frac{I_{r}-I_{i}}{I_{m}}\right]-1=0
$$

and since the discriminant $b^{2}-4 a c>0$ one obtains real solutions, with $\bar{I} \equiv\left(I_{r}-I_{i}\right) / 2 I_{m}$,

$$
\left(\frac{d \beta}{d \alpha}\right)_{ \pm}=-\bar{I} \pm \sqrt{\bar{I}^{2}+1} .
$$

The choice of the sign in (64) is arbitrary, $\beta_{ \pm}^{\prime} \equiv(d \beta / d \alpha)_{ \pm}$can then be substituted into (63a) to obtain $\alpha$ in terms of $\beta$, i.e.,

$$
\alpha_{ \pm}=-\beta \frac{I_{m}+\beta_{ \pm}^{\prime} I_{i}}{I_{r}+\beta_{ \pm}^{\prime} I_{m}} \equiv-\beta f_{ \pm}
$$

which can be used to obtain $\beta$ from (30):

$$
\beta_{ \pm}=\sigma \sqrt{2 l}\left(f_{ \pm}^{2} I_{r}-2 f_{ \pm} I_{m}+I_{i}\right)^{-1 / 2} .
$$

Equations (65) and (66) provide the coefficients in the displacement error function (28) that maximizes the general error integral (26).

Treating the special case $\alpha \neq 0, \beta=0$, that corresponds to maximizing only the real part of (26), one obtains from (62a)

$$
\frac{d \beta}{d \alpha}=-\frac{I_{r}}{I_{m}}
$$

and from (62b)

$$
\left[\int g_{r} g_{i} d x\right]^{2}-\int g_{r}^{2} d x \int g_{i}^{2} d x=0,
$$

which is not, in general, satisfied. Similar results hold for $\alpha=$ $0, \beta \neq 0$; in this case (62a) gives

$$
\frac{d \beta}{d \alpha}=-\frac{I_{m}}{I_{i}}
$$

and (62b) again leads to (68). This shows that these special cases do not maximize, in general, the amplitude of the integral in (26).

\section{APPENDIX II}

\section{The Maximization Procedure for Steered Beams}

In this Appendix the qualitative physical treatment presented in Section III-D is made more precise. Only the essential details are presented.

For steered beams, from Section III-D,

$$
B(x)=b(x) e^{i \epsilon x} .
$$

The error spectrum is then given by

$$
\Delta D(k)=\int\left[b(x+\delta x) e^{i \epsilon(x+\delta x)}-b(x) e^{i \epsilon x}\right] e^{-i k x} d x
$$

where $\delta x(x)$ is subject to constraint (11). The method presented in Section III can be adapted to yield a condition for $\delta x(x)$ that will maximize the real (imaginary) part of (70). For $k=\epsilon$, one obtains for the real part

$\delta x=\frac{1}{2 \eta}\left[b^{\prime}(x+\delta x) \cos (\epsilon \delta x)-\epsilon b(x+\delta x) \sin (\epsilon \delta x)\right]$

corresponding to $(10 \mathrm{c})$. For small $\epsilon \delta x$, the zeroth-order approximation immediately follows; i.e., let $\cos (\epsilon \delta x) \approx 1$, $\sin (\epsilon \delta x) \approx 0$, and $(10 c)$ is recovered. Higher order approximations are obtained by expanding in (71) the nearfield quantities and the trigonometric functions in Taylor and infinite series, respectively, and collecting terms in increasing powers of $\delta x$. Thus,

$$
\begin{aligned}
\delta x=\frac{1}{2 \eta}\left[b^{\prime}(x)+\right. & {\left[b^{\prime \prime}(x)-\epsilon^{2} b(x)\right] \delta x } \\
& \left.+\frac{1}{2}\left[b^{\prime \prime \prime}(x)-\epsilon^{2} b^{\prime}\right](\delta x)^{2}+\cdots\right] .
\end{aligned}
$$

This expression should be compared to the expansion above (12). To first order then,

$$
\delta x=\frac{b^{\prime}(x)}{2 \eta-\left[b^{\prime \prime}(x)-\epsilon^{2} b(x)\right]} .
$$


If

$$
b^{\prime \prime}(x)-\epsilon^{2} b(x) \ll 2 \eta_{1}
$$

where the subscript indicates first order, then the constraint (11) gives

$$
\delta x \cong \sigma_{x} \frac{b^{\prime}(x)}{\left\langle b^{\prime 2}\right\rangle^{1 / 2}}
$$

or

$$
2 \eta_{1}=\frac{1}{\sigma_{x}}\left\langle b^{\prime 2}\right\rangle^{1 / 2}
$$

Again, for small enough $\sigma_{x}$, condition (74) can be satisfied, as well as conditions corresponding to (19a) and (19b). The second-order maximization of the real part of (70), using (75), is

$$
\mu_{2}(\epsilon) \leqslant \frac{1}{\langle b\rangle}\left[\sigma_{x}\left\langle b^{\prime 2}\right\rangle^{1 / 2}+\frac{1}{2} \sigma_{x}^{2} \frac{\left\langle\left(b^{n}-\epsilon^{2} b\right) b^{\prime 2}\right\rangle}{\left\langle b^{\prime 2}\right\rangle}\right] .
$$

This expression corresponds to (21b).

The imaginary part of $(70)$ can be maximized similarly to the above procedure. One obtains

$$
\delta x=\frac{1}{2 \eta}\left[b^{\prime}(x+\delta x) \sin (\epsilon \delta x)+\epsilon b(x+\delta x) \cos (\epsilon \delta x)\right]
$$

and

$$
\delta x \cong \frac{\epsilon b^{\prime}(x)}{2 \eta-\epsilon b^{\prime}(x)}
$$

where, if $2 \eta \Rightarrow \epsilon b^{\prime}(x)$, then from the constraint (11),

$$
2 \eta=\frac{\epsilon}{\sigma_{x}}\left\langle b^{2}\right\rangle^{1 / 2}
$$

and

$$
\delta x \cong \sigma_{x} \frac{b(x)}{\left\langle b^{2}\right\rangle^{1 / 2}} .
$$

The second-order maximization of the imaginary part of (70), using (81), is

$$
\mu_{2}(\epsilon) \leqslant \frac{\epsilon}{\langle b\rangle}\left[\sigma_{x}\left\langle b^{2}\right\rangle^{1 / 2}+\sigma_{x}^{2} \frac{\left\langle b^{\prime} b^{2}\right\rangle}{\left\langle b^{2}\right\rangle}\right] .
$$

To maximize the amplitude of $\Delta D(\epsilon)$, we construct the linear combination

$$
\delta x=\alpha b(x)+\beta b^{\prime}(x)
$$

and use the method outlined in Section III-B and Appendix I to solve for $\alpha$ and $\beta$. This will not be presented here. Finally, the case $k \neq \epsilon$ could be fully developed exactly along the lines presented in this Appendix.

\section{APPENDIX III}

\section{The FAR-Field ERror Spectrum}

In this Appendix the theoretical result in Section III-C is evaluated for the model near field $B(x)=\cos ^{2} \alpha x$, where $2 \alpha \equiv k^{\prime}=\pi / l$. Equation (37) gives the imaginary part of the fractional error, whereas the real part is given by

$$
\left|\frac{\Delta D(k)}{D(0)}\right|^{2} \leqslant \sigma_{x}^{2} \frac{\left\langle B^{\prime 2} \cos ^{2} k x\right\rangle}{\langle B(x)\rangle^{2}} .
$$

When the averages are evaluated one obtains

$$
\begin{aligned}
\left|\frac{\Delta D(k)}{D(0)}\right|^{2} \leqslant \sigma_{x}^{2} \alpha^{2}(1 & \pm \operatorname{sinc} q \\
& \left.\mp \operatorname{sinc}\left(q^{\prime}-q\right) \mp \operatorname{sinc}\left(q^{\prime}+q\right)\right)
\end{aligned}
$$

where $\operatorname{sinc}(q)=\sin (q) / q, q=2 k l$ and $q^{\prime}=2 k^{\prime} l=2 \pi$, and the upper (lower) set of signs give the real (imaginary) part of the fractional error, respectively. These functions of $k$ have been plotted in Fig. 2. Using the error displacement function that gives a maximum error on-axis, the fractional error spectrum as a function of $k$ is given by

$$
\begin{aligned}
\frac{\Delta D(k)}{D(0)} & =\frac{\sigma_{x}}{\langle B\rangle\left\langle B^{\prime 2}\right\rangle^{1 / 2}} \\
\cdot & {\left[\int_{-l}^{l} B^{\prime 2} \cos k x d x+i \int_{-l}^{l} B^{\prime 2} \sin k x d x\right] . }
\end{aligned}
$$

When this is evaluated for $B(x)=\cos ^{2} \alpha x$, one obtains

$$
\begin{aligned}
\left|\frac{\Delta D(k)}{D(0)}\right|^{2}= & 2^{3} \sigma_{x}^{2} \alpha^{2}\left[\frac{1}{2} \operatorname{sinc} \frac{q}{2}\right. \\
& \left.-\frac{1}{4} \operatorname{sinc}\left(q^{\prime}+\frac{q}{2}\right)-\frac{1}{4} \operatorname{sinc}\left(q^{\prime}-\frac{q}{2}\right)\right]^{2}
\end{aligned}
$$

where $q\left(q^{\prime}\right)$ have been defined previously.

In Fig. 3(a) the effects of experimentally induced near field errors on the far field are shown. The maximum error occurs on axis. In Fig. 3(b) the expression (87) is plotted. The qualitative agreement between the experimental and theoretical curves is apparent. For a general (realistic) near field, the error spectrum will be given by a sum over $k^{\prime}$ of functions given in (85) wherein each term is weighted by the spectral component of the square of the derivative of the near field, as can be seen in (37) and (84).

\section{APPENDIX IV}

\section{Radial Displacement Errors in Spherical Scanning}

In this Appendix the equations analyzing $\theta$-displacement errors in Section $V$ are adapted to displacement errors in the radial direction, denoted by $\delta \mathbf{r}(\theta)$. The error displacement function is

$$
\delta \mathbf{r}(\theta)=\delta r(\theta)\left[\left(\frac{x}{R}\right) \hat{x}+\left(\frac{z}{R}\right) \hat{z}\right],
$$




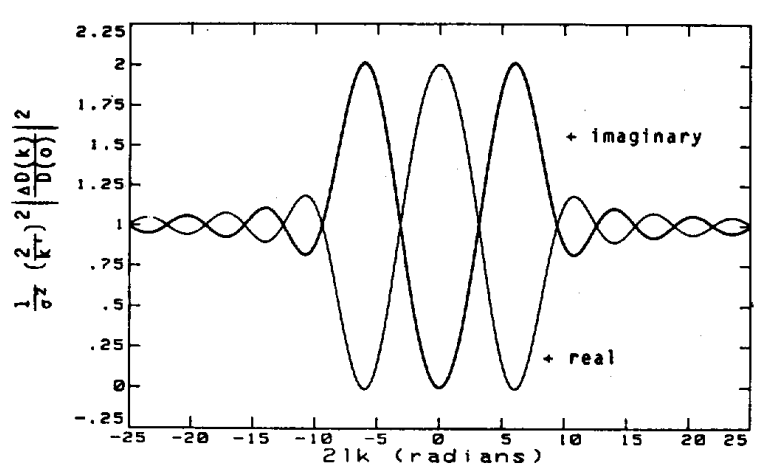

Fig. 2. The amplitude squared of the normalized maximum fractional error as a function of $k$ for a near field $B(x)=\cos ^{2} \alpha x$, where $2 \alpha \equiv k^{\prime}=\pi / l$.

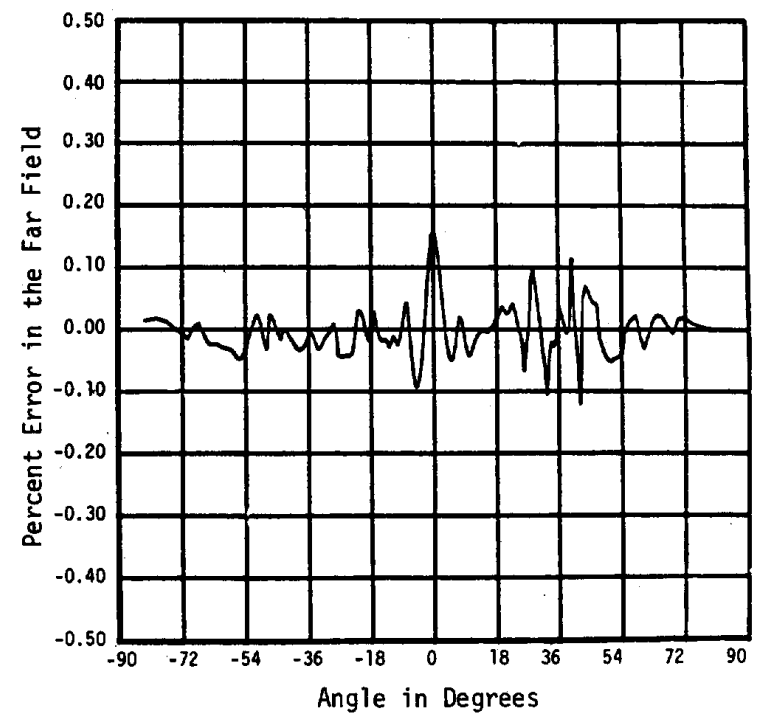

(a)

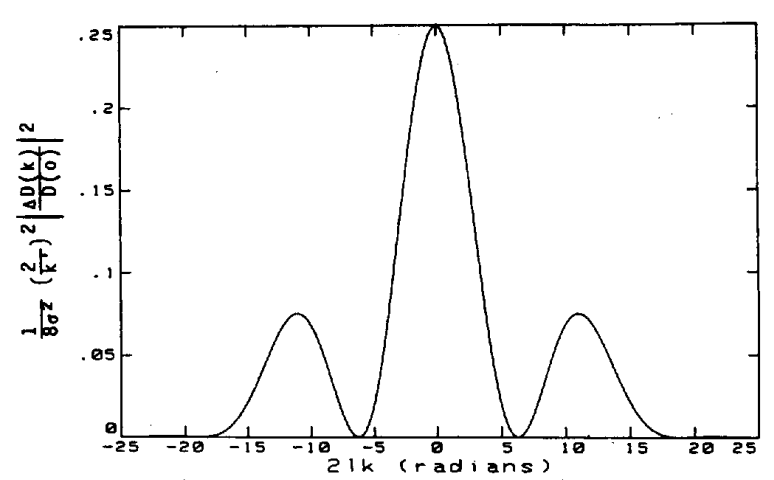

(b)

Fig. 3. (a) Error in percent of maximum amplitude in far-field pattern due to $x$-position errors (same as [3, fig. 17]). (b) Theoretical fractional error in the spectrum when the on-axis error is the maximum. corresponding to (48), and the equation of constraint is

$$
\frac{1}{R \Delta \theta} \int_{L}(\delta r)^{2} \frac{R d \theta}{d x} d x=\sigma_{r}^{2},
$$

corresponding to (49). The expression to be maximized is

$$
\hat{I}=\int_{L}\{B(x+\delta \mathrm{r})-B(x)\} d x-\eta R \int_{L} \frac{(\delta r)^{2}}{z} d x
$$

or

$$
\begin{aligned}
\hat{I}=\int_{L}\left\{\frac{\partial B}{\partial x}\left(\frac{x}{R}\right)+\frac{\partial B}{\partial z}\left(\frac{z}{R}\right)\right\} & \delta r(x) d x \\
& -\eta R \int_{L} \frac{(\delta r)^{2}}{z} d x .
\end{aligned}
$$

Each term is maximized individually by

$$
\delta r(x)=\frac{\sigma_{r}\left(\frac{x}{R}\right)\left(\frac{z}{R}\right) B_{x}(x)}{\left|\left(\frac{z}{R}\right)\left(\frac{x}{R}\right)^{2}\left(B_{x}\right)^{2}\right|_{\theta}^{1 / 2}},
$$

and

$$
\delta r(x)=\frac{\sigma_{r}\left(\frac{z}{R}\right)^{2} B_{z}(x)}{\left|\left(\frac{z}{R}\right)^{3}\left(B_{z}\right)^{2}\right|_{\theta}^{1 / 2}},
$$

corresponding to (53) and (54). The on-axis fractional errors are given by

$$
\mu_{1}^{2}(0) \leqslant \sigma_{r}^{2} \frac{\left\langle\left(\frac{z}{R}\right)\left(\frac{x}{R}\right)^{2}\left(B_{x}\right)^{2}\right\rangle}{\langle B(x)\rangle^{2}},
$$

and

$$
\mu_{1}^{2}(0) \leqslant \sigma_{r}^{2} \frac{\left\langle\left(\frac{z}{R}\right)^{3}\left(B_{z}\right)^{2}\right\rangle}{\langle B(x)\rangle^{2}} .
$$

The amplitude of $\hat{I}$ is maximized by a linear combination of (92) and (93), and an expression similar to (60) and (61) can be written immediately.

\section{ACKNOWLEDGMENT}

Numerous discussions with Allen C. Newell on the previous approaches to the subject area treated in this study are gratefully acknowledged. Useful interaction with Dr. David Hill and Dr. James Randa is also acknowledged.

\section{REFERENCES}

[1] D. M. Kerns, "Plane wave scattering-matrix theory of antennas and antenna-antenna interactions," Nat. Bur. Stand. Monograph 162 , 1981. 
[2] A. D. Yaghjian, "Upper-bound errors in far-field antenna parameters determined from planar near-field measurements. Part 1: Analysis," Nat. Bur. Stand. Tech. Note 667, 1975.

[3] A. C. Newell, "Upper-bound errors in far-field antenna parameters determined from planar near-field measurements. Part 2: Analysis and computer simulation," Lecture notes for Nat. Bur. Stand. Short Course, Antenna Parameter Measurements by Near-Field Techniques, Boulder, CO, 1975, private communication.

44] L. E. Corey, "Analytical compensation for near-field probe positioning errors in calculated far-field antenna patterns," Georgia Inst. Technol., Ph.D. dissertation, May 1981.

[5] L. E. Corey and E. B. Joy, "On computation of electromagnetic fields on planar surfaces from fields specified on nearby surfaces," IEEE Trans. Antennas Propagat, vol. AP-29, no. 2, Mar. 1981.

[6] J. Mathews and R. L. Walker, Mathematical Methods of Physics, 2nd ed.. MA: W. A. Benjamin, Inc., 1970.

[7] A. E. Taylor, Advanced Calculus. New York: Ginn and Company, 1955.
[8] J. B. Conway, Functions of One Complex Variable, 2nd ed. New York: Springer-Verlag, 1984.

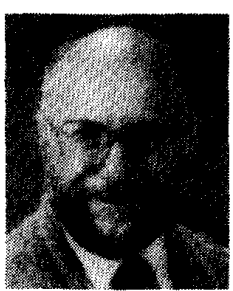

Lorant A. Muth was born in Budapest, Hungary, on November 24,1942 . He received the B.S. degree from the City College of New York, New York, in 1964, the M.S. degree from New York University, New York, in 1966, and the Ph.D. degree from the University of Colorado, Boulder, in 1966, all in physics.

He taught undergraduate physics courses at the State University of New York at Farmingdale, NY, from 1967 to 1972 . He worked in the field of geomagnetism immediately after receiving the Ph.D. degree. Since 1983 he has been a member of the Antenna Metrology Group, Electromagnetic Fields Division at the National Bureau of Standards, Boulder, $\mathrm{CO}$, where he works on theoretical problems pertaining to antenna metrology. 